\title{
SISTEMATIZAÇÃO DA ALTA DE ENFERMAGEM - UMA ANÁLISE FUNDAMENTADA EM ROY
}

\author{
Emanuelle Caires Dias Araújo Nunes ${ }^{1}$, Nilton Alves de Menezes Filho²
}

\begin{abstract}
RESUMO: O estudo objetivou analisar o processo de sistematização da alta hospitalar a partir Modelo de Adaptação de Roy. Trata-se de estudo qualitativo, desenvolvido em hospital público do interior da Bahia com 12 sujeitos entre enfermeiros e pessoas/famílias, delimitados pela saturação dos dados. A coleta, realizada de junho a agosto de 2015, envolveu entrevista semiestruturada e observação não participante da equipe de enfermagem com dados tratados mediante análise temática. Os resultados foram organizados em 05 eixos: avaliação do comportamento da pessoa; avaliação dos estímulos da pessoa; estabelecimento de objetivos para alta; intervenção preparatória para alta; e avaliação do processo de alta. Os eixos foram apresentados em três ângulos: o dito, o executado e o esperado. Conclui-se que ainda é incipiente o planejamento e intervenção da alta, sugerindo aos profissionais a necessidade de sistematização do "Plano de Alta de Enfermagem" a partir de Roy.
\end{abstract}

DESCRITORES: Enfermagem; Planejamento; Alta do paciente; Adaptação; Assistência integral à saúde.

\section{SYSTEMIZATION OF NURSING DISCHARGE - AN ANALYSIS BASED ON ROY}

\begin{abstract}
The objective was to analyze the systemization process of hospital discharge based on Roy's Adaptation Model. A qualitative study was developed at a public hospital in the interior of Bahia, involving 12 subjects, including nurses and people/ families, delimited by the saturation of the data. The data were collected between June and August 2015, involving semistructured interviews and non-participant observation of the nursing team, with data treated through thematic analysis. The results were organized along five axes: assessment of the person's behavior; assessment of the person's stimuli; establishment of objectives for discharge; preparatory intervention for discharge; and assessment of the discharge process. The axes were presented from three angles: what was said, what was done and what was expected. In conclusion, discharge planning and intervention remains incipient, suggesting to the professionals the need to systemize the "Nursing Discharge Plan" based on Roy.
\end{abstract}

DESCRIPTORS: Nursing; Planning; Patient discharge; Adaptation; Comprehensive health care.

\section{SISTEMATIZACIÓN DEL ALTA DE ENFERMERÍA - UN ANÁLISIS FUNDAMENTADO EN ROY}

RESUMEN: El estudio tuvo el objetivo de analizar el proceso de sistematización del alta hospitalar por medio del Modelo de Adaptación de Roy. Es un estudio cualitativo, desarrollado en hospital público del interior de Bahia con 12 sujetos entre enfermeros y personas/ familias, delimitados por la saturación de los datos. Estos fueron obtenidos de junio a agosto de 2015, por medio de entrevista semiestructurada y observación no participante del equipo de enfermería y tratados por análisis temático. Los resultados fueron organizados en 05 ejes: evaluación del comportamiento de la persona; evaluación de los estímulos de la persona; establecimiento de objetivos para alta; intervención preparatoria para alta; y evaluación del proceso de alta. Los ejes fueron presentados en tres ángulos: el dicho, el ejecutado y el esperado. Se concluyó que el planeamiento y la intervención del alta todavía son incipientes, lo que lleva a sugerir a los profesionales la necesidad de sistematización del "Plan de Alta de Enfermería" con base en Roy.

DESCRIPTORES: Enfermería; Planeamiento; Alta del paciente; Adaptación; Asistencia integral a la salud.

${ }^{1}$ Enfermeira. Mestre em Enfermagem. Docente do Instituto Multidisciplinar em Saúde da Universidade Federal da Bahia. Vitória da Conquista, BA, Brasil.

${ }^{2}$ Enfermeiro. Instituto Multidisciplinar em Saúde da Universidade Federal da Bahia. Vitória da Conquista, BA, Brasil.

Autor Correspondente:

Emanuelle Caires Dias Araújo Nunes

Universidade Federal da Bahia

Av. Expedicionários, 20 - 45020-310 - Vitória da Conquista, BA, Brasil

Email: emanuelecdanunes@gmail.com
Recebido: $14 / 03 / 2016$

Finalizado: 08/06/2016 


\section{- INTRODUÇÃO}

A Sistematização da Assistência de Enfermagem (SAE) se dispõe como um aparelho que propicia ordem e direção ao cuidado de Enfermagem. É o instrumento metodológico viabilizador da tomada de decisão clínica, ao representar uma atividade intelectual ordenada e sistemática, baseada em Teorias de Enfermagem, abrangendo o campo interdisciplinar de aparato científico que permite ao profissional desempenhar seu papel com coerência e ética ${ }^{(1-2)}$.

O Conselho Federal de Enfermagem (COFEN), por meio da Resolução $n^{\circ} 358 / 2009$, orienta a inserção da SAE como método organizador do trabalho de enfermagem, operacionalizando o cuidado, tanto no ambiente hospitalar quanto no domiciliar ${ }^{(3)}$. A SAE permite detectar as necessidades de cuidado profissional de cada pessoa, dirigindo a atuação do enfermeiro, para que tenha suas atribuições específicas concretizadas e respeitadas dentro da equipe ${ }^{(4)}$.

Dentre as fases da sistematização, o planejamento se destaca como aquela que confere a base para a práxis de cuidados. Ele pode contribuir para transformar a realidade junto ao indivíduo assistido, ao permitir uma prescrição baseada em evidências. No contexto do planejamento, optou-se pelo recorte do plano de alta, um dispositivo essencial à continuidade do cuidado em domicílio ao encontro da qualidade de vida e prevenção de reinternações.

A adesão ao dispositivo reduz reinternações através do reforço da comunicação, segurança no uso dos medicamentos e melhor compreensão do binômio pessoa-família para gerir o cuidado e as condições próprias que normalmente precipitam readmissão. Assim, incentiva-se o investimento em estudos capazes de definir melhor o papel dos serviços de home-based, tecnologia da informação, apoio ao cuidador e parcerias com a comunidade ${ }^{(5)}$.

O preparo para alta hospitalar deve ser trabalhado a partir da admissão, prevendo encaminhamentos e contato com as unidades de saúde de referência da pessoa para manutenção do cuidado no ambiente domiciliar, demando um planejamento interdisciplinar e integral ${ }^{(6)}$.

Neste sentido, o estudo elege a Teoria de Enfermagem: Modelo de Adaptação de Roy (MAR) como fundamentação ao planejamento sistematizado da alta. Esta teoria compreende a pessoa em reabilitação como um sistema adaptável, que necessita do auxílio da enfermagem para desenvolvimento de respostas positivas, por meio de mecanismos de enfrentamento reguladores e cognatos para adaptação tanto no âmbito hospitalar quanto no domiciliar, englobando família e comunidade ${ }^{(7)}$.

O MAR utiliza a Teoria Geral dos Sistemas na descrição da pessoa como um sistema aberto, ao admitir entradas (estímulos) e saídas (comportamento) que contribuem para sua adaptação(8). Este sistema desenvolve adaptação decorrente da capacidade de respostas positivas das pessoas frente às adversidades. A pessoa mobilizará recursos internos: capacidades, esperanças, sonhos, aspirações, motivações e outros em direção ao domínio. Assim, o MAR compreende a pessoa como um sistema adaptável, em que os estímulos provenientes do ambiente interno e externo ativam mecanismos de resistência para produzir comportamentos saudáveis ${ }^{(7)}$.

A relevância desta investigação incide, para além do explicitado acima, na lacuna identificada por meio de revisão de literatura realizada nas bases da Literatura Latino-Americana e do Caribe em Ciências da Saúde (Lilacs) e Scientific Eletronic Library Online (Scielo), a partir dos descritores: sistematização da assistência de enfermagem, prescrições de enfermagem, plano de alta de enfermagem e Modelo de adaptação de Callista Roy, em um recorte de cinco anos. As evidências revelam uma ampla discussão da $\mathrm{SAE}$, porém, com ênfase prevalente na etapa de prescrição de enfermagem ${ }^{(9-11)}$ em detrimento do planejamento, sobretudo na sua interface com a alta.

Desse modo, emergiram como problemas: "Como se desenvolve o planejamento de enfermagem para a alta hospitalar?" " "Como contribuir para a efetivação deste processo ao encontro da adaptação proposta por Roy?". O objetivo geral foi analisar o processo de sistematização da alta hospitalar a partir Modelo de Adaptação de Roy e específicos: identificar as necessidades percebidas pelo binômio pessoa-família no momento de alta hospitalar, e conhecer a percepção e atuação do profissional enfermeiro acerca deste planejamento na sua prática diária. 
Trata-se de uma pesquisa descritiva e exploratória, de abordagem qualitativa. O cenário abrangeu um hospital público de referência regional, no interior da Bahia. Os sujeitos da pesquisa totalizaram 12 entrevistados: 05 profissionais enfermeiros assistentes do período diurno das unidades de internação médica e cirúrgica da referida instituição, e 07 binômios: pessoas em alta no leito e seus familiares. Ambos os públicos, os quais aceitaram participar da pesquisa, delimitados pela saturação dos dados.

Aos sujeitos foram dados nomes fictícios referentes a tipos de solo e vegetações, respectivamente, para Enfermeiros e Pessoas/Famílias, analogia que compreende ser o tipo de solo (Enfermeiro) fundamental na evolução da vegetação (Pessoa). A coleta foi realizada entre junho e agosto de 2015, por meio de duas entrevistas semiestruturadas com roteiros específicos: um para o enfermeiro e outro para o binômio; e observação não participante do enfermeiro norteado por instrumento capaz de avaliar sua conduta frente à alta da pessoa quanto a: assistência; orientações; planejamento e encaminhamentos realizados. A observação ocorreu no período diurno durante seis semanas consecutivas, sendo realizada até a sua saturação, quanto aos comportamentos observados.

Os resultados foram analisados e categorizados conforme a Análise Temática ${ }^{(12)}$ e organizados em cinco eixos/categorias coincidentes às etapas do MAR, exceto aquela referente aos diagnósticos, adaptação necessária para apresentação coerente dos dados. Deste modo, a entrevista com os enfermeiros gerou "o dito"; a observação dos enfermeiros gerou "o executado" e a entrevista com o binômio gerou "o esperado". A pesquisa foi aprovada por Comitê de Ética e Pesquisa, sob o protocolo n. ${ }^{\circ} 1.001 .259$ de $10 / 03 / 2015$.

\section{RESULTADOS}

Os sujeitos enfermeiros se caracterizaram no grupo etário entre 35 a 52 anos de idade, compondo uma maioria feminina (apenas um homem). Em relação aos sujeitos do binômio paciente-família também predominou o sexo feminino como representante familiar acompanhante (apenas dois homens), envolvendo a faixa etária de 24 a 62 anos, com procedência mista entre o município do hospital e outros municípios.

Os resultados seguem apresentados em cinco eixos: avaliação da pessoa, avaliação dos estímulos da pessoa, estabelecimento de objetivos para alta, intervenção preparatória para alta e avaliação do processo de alta. Cada um destes está organizado em três perspectivas: o dito (oriundo das entrevistas com o enfermeiro); o executado (apreendido a partir da técnica de observação dos enfermeiros) e o esperado (procedente das respostas da pessoa e sua família).

Para melhor compreensão, os resultados seguem apresentados em tabelas que permitem a visualização e comparação entre dito-executado-esperado em cada eixo supracitado.

\section{Eixo 1: Avaliação do comportamento da pessoa}

A Tabela 1 apresenta no dito os dados que o enfermeiro refere como importantes no processo de avaliação do comportamento, os quais não foram observados pelo pesquisador no executado, embora perspectivados no esperado pela pessoa.

\section{Eixo 2: Avaliação dos estímulos da pessoa}

A Tabela 2 apresenta no dito as ações que o enfermeiro considera ideais no processo de avaliação dos estímulos, as quais não foram observadas pelo pesquisador no executado, seguido da apresentação, no esperado, das necessidades de estímulos apoiadores do binômio. 


\section{Eixo 3: Estabelecimento de objetivos para alta}

A Tabela 3 apresenta os objetivos para alta, traçados pelo enfermeiro, nem sempre expressos junto à pessoa, e em seguida os objetivos verbalizados pela família.

\section{Eixo 4: Intervenção preparatória para alta}

A Tabela 4 apresenta no dito, executado e esperado as intervenções ideais versus as realizadas em detrimento das almejadas.

\section{Eixo 5: Avaliação do processo de alta}

A Tabela 5 apresenta avaliação do processo de alta bem como suas lacunas e inquietações que perpassam tanto o profissional como a pessoa convalescente.

Tabela 1 - Avaliação do comportamento da pessoa. Vitória da Conquista, BA, Brasil, 2015

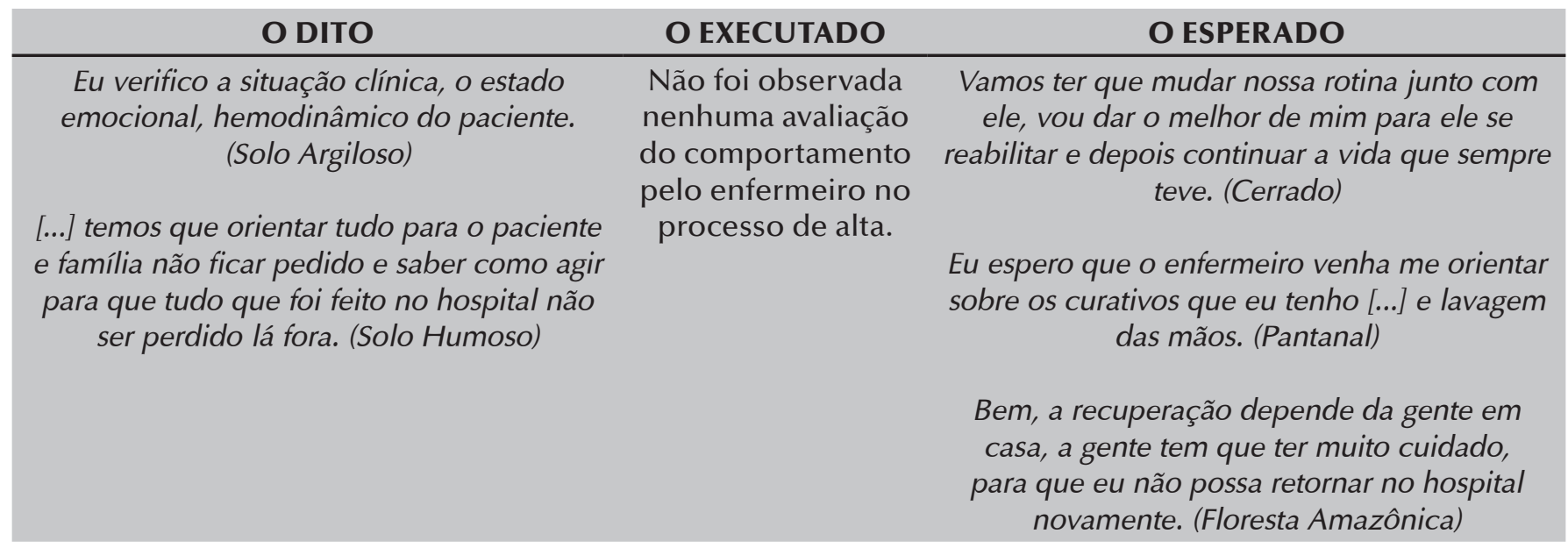

Tabela 2 - Avaliação dos estímulos da pessoa. Vitória da Conquista, BA, Brasil, 2015

\begin{tabular}{|c|c|c|}
\hline O DITO & O EXECUTADO & O ESPERADO \\
\hline $\begin{array}{r}\text { O papel do enfermeiro na alta é avaliar em } \\
\text { que condições este paciente está saindo de } \\
\text { alta, em termos de recuperação e reabilitação } \\
\text { dele. (Solo Arenoso) } \\
\text { Quando temos tempo disponível, eu sempre } \\
\text { gosto de orientar principalmente pacientes } \\
\text { diabéticos que saem de alta, sobre os } \\
\text { cuidados com o uso da alimentação [...] se } \\
\text { ele não faz esse tratamento lá fora ele vai } \\
\text { acabar retornando.(Solo Humoso) } \\
\text { Se o pessoa saiu da unidade com alguma } \\
\text { escara [...] orientar os familiares quanto ao } \\
\text { cuidado [...] medicação, retorno, curativo, } \\
\text { e acompanhamento com especialista. (Solo } \\
\text { Calcário) }\end{array}$ & $\begin{array}{l}\text { Não foi observada } \\
\text { nenhuma avaliação } \\
\text { de estímulos por } \\
\text { parte da equipe de } \\
\text { enfermagem. }\end{array}$ & $\begin{array}{c}\text { Eu queria que o enfermeiro esclarecesse } \\
\text { minhas dúvidas. Eu moro com ele sozinha, ele } \\
\text { depende de mim para tudo, e profissionais de } \\
\text { saúde vão uma vez por mês em minha casa } \\
\text { [...]. Eu queria que ele me orientasse de como } \\
\text { movimentar ele, pois ele tem corpo de adulto e } \\
\text { eu não aguento [...]. (Campos Sulinos) } \\
\\
\text { [...] quando me falaram que poderia ser } \\
\text { meningite eu fiquei muito preocupada [...] mas } \\
\text { os profissionais foram me esclarecendo sobre } \\
\text { os procedimentos. (Mata Atlântica) }\end{array}$ \\
\hline
\end{tabular}




\begin{tabular}{|c|c|c|}
\hline O DITO & O EXECUTADO & O ESPERADO \\
\hline $\begin{array}{c}\text { O paciente na alta hospitalar tem que sair } \\
\text { daqui completamente orientado em relação } \\
\text { a tudo que ele vá depender do serviço de } \\
\text { saúde, até mesmo o caso do retorno. (Solo } \\
\text { Humoso) } \\
\text { É necessário que façamos a devida } \\
\text { orientação junto ao paciente, e familiares } \\
\text { para que tenha continuidade em casa e } \\
\text { alcance a recuperação ideal. Isso inclui } \\
\text { retorno do paciente e continuidade do } \\
\text { tratamento terapêutico [...]. (Solo Arenoso) } \\
\text { O enfermeiro deve dar orientações de todos } \\
\text { os cuidados que esse paciente tem que ter } \\
\text { em casa. [...] curativos, alimentação, uso } \\
\text { correto da medicacão [... (Solo Ácrico) }\end{array}$ & $\begin{array}{l}\text { O enfermeiro e } \\
\text { sua equipe não } \\
\text { verbalizam junto } \\
\text { ao paciente suas } \\
\text { metas para a alta e } \\
\text { também não fazem } \\
\text { nenhum registro } \\
\text { neste sentido no } \\
\text { prontuário. }\end{array}$ & $\begin{array}{l}\text { Que ele melhore cada vez mais faço tudo para } \\
\text { que ele tenha saúde. (Mata de Araucária) } \\
\text { Estar sempre cuidando da saúde, pois eu ficava } \\
\text { focada no trabalho e na parte da saúde era } \\
\text { negligente. (Mata Atlântica) } \\
\text { Cuidar de mim, alimentar bem, não fazer } \\
\text { coisas que me prejudiquem. (Caatinga) }\end{array}$ \\
\hline
\end{tabular}

Tabela 4 - Intervenção preparatória para alta. Vitória da Conquista, BA, Brasil, 2015

\begin{tabular}{ccc} 
O DITO & O EXECUTADO & O ESPERADO \\
\hline $\begin{array}{c}\text { Retirar acesso periférico, central. Orientar } \\
\text { a família quanto aos cuidados com } \\
\text { dispositivos. (Solo Calcário) }\end{array}$ & $\begin{array}{c}\text { Somente foram } \\
\text { executadas: retirada } \\
\text { de dispositivos do } \\
\text { paciente. }\end{array}$ & $\begin{array}{c}\text { Eu queria ser melhor orientada de como lidar } \\
\text { com ele, aprender alguma forma de fazer uma } \\
\text { fisioterapia nele. (Mata de Araucária) }\end{array}$ \\
$\begin{array}{c}\text { Normalmente nós orientamos com relação } \\
\text { aos cuidados com medicação, alimentação e } \\
\text { o ambiente dependendo da patologia. (Solo } \\
\text { Argiloso) }\end{array}$ & $\begin{array}{c}\text { Na minha opinião, fazer um relatório. Procurar } \\
\text { saber como será minha saída do hospital. } \\
\text { (Campos Sulinos) }\end{array}$ \\
& $\begin{array}{c}\text { Eu espero orientações do enfermeiro sobre os } \\
\text { remédios que tenho que tomar [...] o repouso } \\
{[. . .] \text { meu tratamento. Isso é muito significativo }} \\
\text { [...] ajuda muito. (Cerrado) }\end{array}$
\end{tabular}

Tabela 5 - Avaliação do processo de alta. Vitória da Conquista, BA, Brasil, 2015

\begin{tabular}{ccc} 
O DITO & O EXECUTADO & O ESPERADO \\
\hline $\begin{array}{c}\text { Ao meu ver, está sendo pouco efetiva, porque } \\
\text { não é uma orientação completa. (Solo Ácrico) }\end{array}$ & $\begin{array}{c}\text { Não foi observada } \\
\text { nenhuma forma de } \\
\text { avaliação da alta. }\end{array}$ & $\begin{array}{c}\text { Ruim, pois eu perguntava as coisas e ele não } \\
\text { me informava [...] Fui pedir o relatório ao } \\
\text { enfermeiro e ele nem sabia que o paciente } \\
\text { estava de alta. Eu não fui orientada sobre } \\
\text { nada. (Mata de Araucária) }\end{array}$ \\
$\begin{array}{c}\text { [...] a rotina é diferente do que deveria ser na } \\
\text { teoria. Infelizmente não é uma coisa perfeita, } \\
\text { porque a depender do momento da correria } \\
\text { da clínica, não tem como fazer as orientações } \\
\text { [...]. (Solo Humoso) }\end{array}$ & $\begin{array}{c}\text { Depois que eu recebi a alta o enfermeiro não } \\
\text { veio aqui, quem veio foi médico. (Cerrado) }\end{array}$ \\
$\begin{array}{c}\text { Mediante as condições de trabalho que a } \\
\text { gente tem, eu acho razoavelmente satisfatória } \\
\text { nossa conduta frente à alta hospitalar. (Solo } \\
\text { Arenoso) }\end{array}$ & $\begin{array}{c}\text { Bom, pois eu tive atenção dos profissionais [...] } \\
\text { da Residente de Enfermagem que me ajudou } \\
\text { muito. (Mata Atlântica) }\end{array}$ \\
\end{tabular}




\section{- DISCUSSÃO}

A avaliação do comportamento apresentado na Tabela 1 representa o primeiro eixo do MAR. Tal avaliação deve consistir na coleta dos dados da pessoa, com intuito de obter o maior número de informações viabilizadoras de uma intervenção singular às necessidades de saúde do indivíduo. Envolveria a avaliação de todas as respostas comportamentais que esta pessoa demonstra ou refere por meio das capacidades de observação, medição e entrevista ${ }^{(7)}$.

Infelizmente, os resultados demonstram incipiência da avaliação do comportamento no cenário do estudo, e, embora exista inferência por parte do enfermeiro em verificar aspectos do comportamento no "dito", isso não foi observado no "executado", o que resulta em um "esperado" pelo binômio pessoa/ família expressivo em lacunas, as quais podem ser preenchidas por uma avaliação de enfermagem capaz de reforçar comportamentos adaptativos e reorientar aqueles ineficazes.

A investigação comportamental perpassa por quatro mecanismos adaptativos: fisiológico, autoconceito, função do papel e interdependência. Isso trará uma abordagem sistemática e integral da pessoa, propiciando uma atenção qualificada à melhor condução do processo de alta hospitalar ${ }^{(7)}$. Deste modo, o MAR norteia a investigação em uma perspectiva orientadora, possibilitando o levantamento de problemas pela análise do comportamento, identificando diagnósticos norteadores das intervenções ${ }^{(13)}$.

Esta etapa investigativa direciona o profissional a estabelecer prioridades diante de comportamentos ineficazes, para então identificar os estímulos pessoais capazes de mobilizar o comportamento adaptativo.

O segundo eixo consiste na avaliação dos estímulos. Entende-se por estímulo aquilo que provoca uma resposta e esclarece a etiologia do problema. O estímulo é um elemento central no MAR. A pessoa, enquanto sistema, é compreendida pela capacidade de se adaptar e criar mudanças no meio ambiente a partir dos estímulos, que podem ser de três tipos: focal, que é o estímulo interno ou externo que constitui o maior grau de mudança, gerando um forte impacto; contextual, que envolve todos os outros estímulos presentes na situação que contribuem para o efeito do estímulo focal; e residual, que representa os fatores cujos efeitos na situação atual não são centrais, podendo ser inconscientes para a pessoa, ou seja, fatores descentralizados da situação atual, mas que a influenciam. A vivência dos estímulos é simultânea, ocorrendo sem ordem aparente, de modo que a pessoa nem sempre os identifica ${ }^{(14)}$. Sua avaliação se constitui em um dos mais evidentes diferenciais do Processo de Enfermagem proposto por Roy e se mostra ainda mais relevante no procedimento da alta.

No entanto, a Tabela 2 expressa uma incipiente referência no "dito" que revela pouca familiaridade dos enfermeiros com a investigação dos estímulos, acrescida da ausência de adesão por parte deste profissional observada no "executado", em meio à eloquente expressão do binômio referente à sua necessidade: o "esperado". Eles desejam sair seguros sobre como lidar com a nova situação, de modo a assumir/contribuir para um comportamento adaptativo, o que é muitas vezes frustrado devido à falta de orientação por parte dos profissionais.

Neste contexto, o planejamento da alta hospitalar representa uma oportunidade para o cuidado continuado direto e indireto ao binômio em seu domicílio no pós-alta através da educação em saúde ${ }^{(15)}$. Afinal, a família constitui um estímulo importante, que, por meio de suas estruturas e relacionamentos, pode desenvolver ou revelar algum tipo de comportamento ineficaz ou adaptativo. Com isso, é necessário que o enfermeiro detecte essas características familiares e as integre na dinâmica do processo saúde-doença, a fim de propiciar melhor qualidade de vida, sobretudo no ambiente domiciliar.

A avaliação de estímulos presentes versus as necessidades de cuidados também deve revelar os fatores de risco simples, como a compreensão de medicamentos, as limitações do estado funcional, entre outras demandas ${ }^{(5)}$. O enfermeiro deve ser capaz de detectar esses estímulos junto ao binômio e contribuir com melhores resultados nas adaptações, ao interpretar os dados no direcionamento dos diagnósticos de enfermagem norteadores dos objetivos a serem estabelecidos.

O estabelecimento de objetivos para alta é feito a partir dos diagnósticos de enfermagem levantados a partir da avaliação do comportamento e dos estímulos. As metas são os comportamentos finais que 
a pessoa deve atingir e devem incluir: comportamento, mudança esperada e estrutura de tempo. Assim, as metas de longo prazo representam a resolução de problemas adaptativos, e as metas de curto prazo identificam os comportamentos esperados da pessoa após o controle de estímulos focais e contextuais ${ }^{(7)}$.

Os resultados expostos na Tabela 3 demonstram que os enfermeiros possuem como objetivo a orientação completa junto ao binômio de como manter ou melhorar a sua saúde após alta, entretanto o "dito" não focaliza os comportamentos esperados do binômio, mas apenas o comportamento esperado do próprio enfermeiro. De fato, este não executa a formulação de suas metas - "o executado" - e, do outro lado, o binômio expressa no "esperado" o comportamento que eles esperam assumir na continuidade da recuperação da saúde em domicílio, remetendo à equipe a responsabilidade de participar ativamente da construção deste momento.

É relevante que as metas postas sejam instituídas em conjunto com a pessoa e seus familiares, bem como o esclarecimento de todas as dúvidas, pois a pessoa/família que esteja ativamente envolvida neste processo terá maior probabilidade de alcançar seus objetivos ${ }^{(7)}$.

Neste contexto, o cuidado de enfermagem, visualizado a partir do MAR, possibilita traçar metas direcionadas ao restabelecimento da adaptabilidade saudável, sendo possível relacioná-las às intervenções necessárias a um trabalho de efetivo desempenho ao encontro da pessoa que recebe o cuidado, considerando-a no todo em suas relações com ambiente interno e externo ${ }^{(16)}$.

Assim, pode-se inferir que o não estabelecimento de metas pelo enfermeiro compromete significativamente a recuperação da pessoa convalescente, o que alerta para a necessidade de mudanças quanto ao estabelecimento de objetivos junto à pessoa/família. Este é o caminho viável à sistematização de estratégias baseadas em evidências e orientações efetivas capazes de ampliar as possibilidades de o binômio atingir as metas no ambiente domiciliar.

O eixo quatro refere-se à intervenção, fase do Processo de Enfermagem que confere o cuidado propriamente dito ao binômio, bem como a execução do que foi estabelecida como prescrição de enfermagem a fim de promover a adaptação da pessoa em sua realidade, a partir de seus mecanismos reguladores internos e externos ${ }^{(1,7)}$.

A Tabela 5 evidencia um processo de intervenção desorganizado dos enfermeiros frente à alta. A atuação da equipe se mostra restrita à simples retirada de dispositivos invasivos no momento da alta, às vezes acrescida de orientações simplórias e gerais para a pessoa convalescente. Com isso, as atividades executadas não vão ao encontro das necessidades identificadas na avaliação do comportamento e dos estímulos, perdurando as adaptações ineficazes e a carência do binômio tão evidente no "esperado".

Diante disso, a família, também referida como cuidadora informal, assume a responsabilidade de buscar informações e estabelecer um diálogo com os serviços formais de saúde, o que consegue apenas com uma grande dose de engenhosidade. Eles têm que dedicar esforços incansáveis e persistência para gerenciar a complexidade dos serviços de saúde e alcançarem os conhecimentos e orientações necessários à adequada tomada de decisão durante e após a alta ${ }^{(17)}$.

Deste modo, a implementação de enfermagem, aqui compreendida como cuidado formal, deve dialogar com o binômio para ampliar a capacidade de enfrentamento pessoal, na medida em que seus estímulos totais permaneçam adaptados ${ }^{(7)}$, apontando para o desfecho do Processo de Enfermagem frente à alta e a devida avaliação do cuidado realizado.

O eixo cinco consiste na avaliação do processo de alta e coincide com a última etapa do MAR. Nesta fase, as metas são comparadas de acordo com os mecanismos de entrada (intervenção), momento em que o profissional detectará se a pessoa conseguiu obter as metas estabelecidas ou se afastou delas ${ }^{(7)}$.

O que pode se observar nos resultados da Tabela 5 é que a avaliação do processo de alta hospitalar no cenário e público pesquisado é inconsistente. Esta constatação era previsível pela incipiência das etapas anteriores. Infelizmente, o "executado" não demonstra nenhuma evidência de avaliação da alta pela enfermagem, o que é assumido pelo enfermeiro no "dito" sobre o processo e refletido no "esperado" no qual o binômio expressa eloquentemente o desapontamento com o plantonista de enfermagem frente à alta. 
Na avaliação o enfermeiro deve lançar mão de suas qualidades de: observação; mediação e entrevista, julgando as intervenções ao refletir sobre as metas atingidas e necessidades de readequações para que a pessoa obtenha o resultado esperado para aquele diagnóstico estabelecido ${ }^{(7)}$. A práxis do Processo de Enfermagem proporciona à pessoa segurança em relação à assistência prestada, resultando numa melhor compreensão do seu problema, favorecendo a recuperação e manutenção da saúde ${ }^{(18)}$.

Portanto, após a alta hospitalar ser prescrita, o enfermeiro deverá prover ao binômio pessoa/ família uma assistência sistematizada e segura para a continuidade do cuidado no âmbito domiciliar. Entretanto, a incipiente adesão dos enfermeiros a esta práxis contribui para possível recorrência ou agravamento da patologia, provocando reinternações e descontinuidade da saúde.

\section{CONCLUSÃO}

A pesquisa alcançou o objetivo traçado, ressaltando-se a enriquecedora contribuição do referencial teórico-metodológico trazido por Roy. A partir dos subsídios da teoria, pode-se observar que os profissionais de enfermagem cultivam um comportamento simplista com relação ao seu papel diante da alta hospitalar, afetando a sua relação de cuidado ao binômio no que tange à promoção da sua saúde e qualidade de vida.

A relação entre os eixos revela discordância entre o que é relatado como prática e o que é implementado efetivamente, comprometendo a efetividade do planejamento frente à alta hospitalar e o restabelecimento da saúde pessoa.

Diante disso, observa-se a imperiosa necessidade da adesão por parte da enfermagem à SAE para a alta hospitalar, sugerindo-se o MAR como teoria propícia ao aperfeiçoamento do planejamento da alta, oportunizando ampliar a visão e atuação profissional frente às necessidades do binômio no domicílio. Incentiva-se, então, a adesão ao cuidado alicerçado pela SAE e pelas Teorias de Enfermagem como possibilidade inovadora do seu processo de trabalho, no direcionamento da valorização desta profissão.

Nessa perspectiva, este estudo sugere a criação de um instrumento norteador para sistematização da alta hospitalar, inspirado no MAR, no qual o profissional terá oportunidade de dispor seus conhecimentos em prol de uma reabilitação continuada da pessoa. Este poderá ser composto por: identificação pessoal; descrição de metas específicas (construídas conjuntamente com o binômio, a partir do levantamento de comportamentos e estímulos da pessoa e dos consequentes diagnósticos); prescrições de enfermagem; orientação para execução da prescrição médica; encaminhamentos e retorno; assinatura, carimbo e contato profissional do enfermeiro.

A adesão ao plano de alta sistematizado conferirá importante diferencial à enfermagem, elevando sua participação frente ao processo saúde-doença, oportunizando a inovação desta profissão ao resgatar saberes-fazeres necessários para uma atuação qualificada à sociedade.

\section{REFERÊNCIAS}

1. Potter PA, Perry AG. Fundamentos de Enfermagem. 8a ed. Rio de Janeiro: Elsevier; 2013.

2. Harkreader H, Hogan MA, Thobaben M. Fundamentals of nursing: caring and clinical judgment. $3^{\mathrm{a} e d .}$ Philadelphia, Pennsylvania,USA: W.B Saunders Company; 2007.

3. Conselho Federal de Enfermagem (COFEN). Resolução n. 358, de 15 de outubro de 2009. Dispõe sobre a Sistematização da Assistência de Enfermagem e a implementação do Processo de Enfermagem em ambientes, públicos ou privados, em que ocorre o cuidado profissional de Enfermagem, e dá outras providências. [Internet] Brasília:COFEN; 2009 [acesso em 15 nov 2015]. Disponível: http://www.cofen.gov.br/resoluo-cofen-3582009_4384. html.

4. Maria MA, Quadros FAA, Grassi MFO. Sistematização da assistência de enfermagem em serviços de urgência e emergência: viabilidade de implantação. Rev. bras. enferm. [Internet] 2012; 65(2) [acesso em 28 mar 2016]. 
5. Kripalani S, Theobald CN, Anctil B, Vasilevskis EE. Reducing hospital readmission rates: current strategies and future directions. AnnuRev Med. [Internet] 2014; (65)[acesso em 15 jul 2015]. Disponível:http://dx.doi.org/10.1146/ annurev-med-022613-090415.

6. Cheade MFM, Frota OP, Loureiro MDR, Quintanilha ACF. Residência multiprofissional em saúde: a busca pela integralidade. CogitareEnferm. [Internet] 2013; 18(3) [acesso em 18 mai 2015].Disponível:http://dx.doi.org/10.5380/ ce.v18i3.33577.

7. Roy C, Andrews HA. Teoria da Enfermagem: O Modelo de Adaptação de Roy. Porto Alegre: Piaget; 1999.

8. George JB, colaboradores. Teorias de Enfermagem: Os fundamentos à prática profissional. Porto Alegre: Artmed; 2000.

9. da Cruz AMP, Almeida MA. Competências na formação de Técnicos de Enfermagem para implementar a Sistematização da Assistência de Enfermagem. Rev. esc. enferm. USP. [Internet] 2010; 44(4) [acesso em 28 mar 2016]. Disponível: http://dx.doi.org/10.1590/S0080-62342010000400009.

10. Silva EGC, de Oliveira VC, Neves GBC, Guimarães TMR. O conhecimento do enfermeiro sobre a Sistematização da Assistência de Enfermagem: da teoria à prática. Rev. esc. enferm. USP. [Internet] 2011; 45(6) [acesso em 28 mar 2016]. Disponível: http://dx.doi.org/10.1590/S0080-62342011000600015.

11. LuziasMF, VicotrMAG, Lucena AF. Diagnóstico de enfermagem Risco de quedas: prevalência e perfil clínico de pacientes hospitalizados. Rev. Latino-Am. Enfermagem. [Internet] 2014; 22(2) [acesso em 20 jan 2016]. Disponível: http://dx.doi.org/10.1590/0104-1169.3250.2411.

12. Minayo MCS. O desafio do conhecimento: pesquisa qualitativa em saúde. São Paulo: Hucitec; 2007.

13. Coelho SMS, Mendes IMDM. Da pesquisa à prática de enfermagem aplicando o modelo de adaptação de Roy. Esc. Anna Nery. [Internet] 2011; 15(4) [acesso em 27 jul 2014]. Disponível: http://dx.doi.org/10.1590/S141481452011000400026.

14. Roy SC, Andrews HA. Teoria da enfermagem:o modelo de adaptação de Roy. Lisboa: Instituto Piaget; 2001.

15. Delatorre GP, Sá SPC, Valente GSC, Silvino ZR. Planejamento para a alta hospitalar como estratégia de cuidado de enfermagem: revisão integrativa. Revenferm UFPE online. [Internet] 2013; 7(n.esp) [acesso em 29 jun 2014]. Disponível: http://www.revista.ufpe.br/revistaenfermagem/index.php/revista/article/download/3968/8170.

16. Costa IKF, da Nóbrega WG, Costa IKF, Torres GV, Lira ALBC, Tourinho FSV, et al. Pessoas com úlceras venosas: estudo do modo psicossocial do Modelo Adaptativo de Roy. Rev. Gaúcha Enferm. 2011; 32(3) [acesso em 29 jun 2014]. Disponível: http://dx.doi.org/10.1590/S1983-14472011000300018.

17. Bragstad LK, Kirkevold M, Foss C. The indispensable intermediaries: a qualitative study of informal caregivers' struggle to achieve influence at and after hospital discharge. BMC Health Serv. Res. 2014; 14(331) [acesso em 28 jul 2015]. Disponível: http://dx.doi.org/10.1186/1472-6963-14-331.

18. de Medeiros AL, dos Santos SR, Cabral RWL. Sistematização da assistência de enfermagem na perspectiva dos Enfermeiros: uma abordagem metodológica na teoria fundamentada. Rev. Gaúcha Enferm. [Internet] 2012; 33(3) [acesso em 02 jun 2014]. Disponível: http://dx.doi.org/10.1590/S1983-14472012000300023. 